\title{
Synergy of microcapsule polysaccharides and Bacillus subtilis on the growth, immunity and resistance of sea cucumber Apostichopus japonicus against Vibrio splendidus infection
}

\author{
Ying Fan $\cdot$ Xiaoqing $\mathrm{Yu} \cdot \mathrm{La} \mathrm{Xu} \cdot$ Shuxian Wang $\cdot$ \\ Haibin Ye $\cdot$ Jing Diao $\cdot$ Xiusheng Yang $\cdot$ \\ Tianbao Li
}

Received: 29 November 2012/ Accepted: 31 May 2013/Published online: 18 July 2013

(c) The Author(s) 2013. This article is published with open access at Springerlink.com

\begin{abstract}
A 4-week feeding trial was conducted to determine the effects of different dietary supplements on the growth, immunity and resistance of sea cucumber Apostichopus japonicus against Vibrio splendidus infection. The control group was supplied with blank microcapsules, and Astragalus polysaccharide (APS) microcapsules, tuckahoe polysaccharide (TPS) microcapsules, (APS + TPS) microcapsules, (APS + TPS) microcapsules + Bacillus subtilis, were tested for effects. Coelomic fluid was collected at 7-day intervals to test activities of lysozyme (LSZ), superoxide dismutase (SOD), alkaline phosphatase (AKP), and complement 3 (C3) content. After the feeding trial, the specific growth rate of sea cucumbers fed a diet supplemented with (APS + TPS) microcapsules $+B$. subtilis was significantly increased $(P<0.05)$; activities of LSZ, SOD, AKP and C 3 content were significantly higher than in other groups $(P<0.05)$. The challenge test showed that the cumulative mortality of sea cucumbers fed a diet supplemented with (APS + TPS) microcapsules $+B$. subtilis reduced significantly $(P<0.05)$. In conclusion, dietary combinations of (APS + TPS) microcapsules + B. subtilis has a potential for use in diet formulations for sea cucumbers to significantly increase growth, immunity and disease resistance against $V$. splendidus infection.
\end{abstract}

Y. Fan $\cdot$ X. Yu $\cdot$ L. Xu $\cdot$ S. Wang $\cdot$ H. Ye $\cdot$ J. Diao $\cdot$ X. Yang

T. Li (ه)

Mariculture Institute of Shandong Province,

Shandong Province Key Laboratory for Disease Control

in Mariculture, No. 47 Guizhou Road, Qingdao 266002,

Shandong, China

e-mail: 1tb1601@126.com

Y. Fan

e-mail: fy_fy123@126.com
Keywords Apostichopus japonicus · Polysaccharide · Bacillus subtilis · Growth · Immunity · Vibrio splendidus

\section{Introduction}

As a traditional food and invigorant, sea cucumber is nowadays extensively cultivated in China, which also brings severe diseases. That's why antibiotics and chemotherapeutics are used today. However, abuse of these results in the spread of drug-resistant pathogens, environmental pollution and unexpected residues in aquaculture [1-4]. As an echinoderm species, sea cucumbers lack an adaptive immune system, and its key defenses against different substances are cellular and humoral immune responses. So the most promising method for controlling sea cucumber disease in aquaculture is to strengthen its defense mechanisms by prophylactic administration of immunostimulants [5].

Many Chinese herbs, such as Astragalus membranaceus and tuckahoe, have been used as immune boosters for nearly 2000 years [6-9]. Many feeding trials and other tests have shown that polysaccharides have significant immunostimulatory effects through different mechanisms, for example by activating mouse B cells and macrophages, regulating the intestinal microbiota to improve productive performance, as well as enhancing the nonspecific immunity of some animals, such as lysozyme, superoxide, and alkaline phosphatase activities in fish or A. japonicus [1013]. But application of microcapsule technology in polysaccharides has not been investigated, and has not been applied in A. japonicus, either.

Most researchers applied only Chinese herb or Bacillus as immunostimulants in their studies [13-15] and a few of them have combined different kinds of Chinese herbs or 
active polysaccharides or Bacillus alone in order to amplify the immune response of aquatic animals [16-18], but their synergistic effects have not been confirmed. In previous work we found that there was better function by injecting Astragalus polysaccharide (APS) and tuckahoe polysaccharide (TPS) into sea cucumber, and APS enhanced the immunity of A. japonicus [19]. In the present study, microcapsule technology can decrease the problem of dissolving of polysaccharides, and polysaccharides can be directly fed in aquaculture. To the best of our knowledge, effects of (APS + TPS) microcapsules $+B$. subtilis supplementation on growth, immune responses and disease resistance of sea cucumber have not been defined.

\section{Materials and methods}

Experimental animals and culture condition

Healthy sea cucumbers (initial weight $40.2 \pm 2.0 \mathrm{~g}$, mean $\pm \mathrm{SE}$ ) were obtained from a farm in Qingdao (China) and kept in cylindrical 61 tanks with recirculating seawater for a 2-week conditioning period. During the experiment, the seawater temperature was $15-18{ }^{\circ} \mathrm{C}, \mathrm{pH}$ was 7.8-8.2, salinity was 31-32 PSU, dissolved oxygen was $>5 \mathrm{mg} / \mathrm{l}$. One-half of the seawater in the recirculating system was replaced by fresh seawater once per day and all was replaced once per week to maintain the water quality.

\section{Experimental design and diets}

Sea cucumbers were randomly divided into six groups, three replicates per group and 12 sea cucumbers per replicate. The basal diet with dried seaweed Sargassum thunbergii meal (group 1) was fed superfluously at a rate of $2 \%$ body weight; the basal diet was supplemented with blank microcapsules (group 2), APS microcapsules (group 3), TPS microcapsules (group 4), (APS + TPS) microcapsules (group 5), (APS + TPS) microcapsules $+B$. subtilis (group 6). These supplements were fed at a rate of $3 \%$ basal diet. All experimental animals were fed with their different diets at 16:00 hours.

APS and TPS were extracted from Chinese herbs Astragalus membranaceus and tuckahoe by the method of water decoction. The polysaccharide content of APS was up to $37 \%$ and TPS was $50 \%$. Microcapsules were prepared through the spray method of "tiny hole and solidifying bath", the encapsulation rate was up to $85 \%$ and the loading dose was $17 \%$. For $B$. subtilis, the number of living bacteria was $10^{11} \mathrm{CFU} / \mathrm{g}$, bought from Qingdao Biocom Biology Technology Company, and added at a rate of $2 \times 10^{7} \mathrm{CFU} / \mathrm{g}$ body weight.
Experimental procedure and sampling procedure

Three individuals in each replicate were randomly sampled for coelomic fluid on 7th, 14th, 21st, and 28th days. Coelomic fluid was collected with a 1-ml sterile syringe through the body wall and was freeze-thawed again. For serum separation, the collected coelomic fluid was spun down at 4,000 rpm for $10 \mathrm{~min}$ at $4{ }^{\circ} \mathrm{C}$. The supernatant was stored in sterile microcentrifuge tubes at $-20{ }^{\circ} \mathrm{C}$ for use. At the end of the 4 -week feeding trial, the remaining sea cucumbers were weighed to monitor growth, and challenged against $V$. splendidus.

Specific growth rate (SGR)

The SGR is an important factor for measuring growth. We monitored it by collectively weighing the sea cucumbers in each group. The growth was calculated by the formula: specific growth rate $(\mathrm{SGR})=\left(\ln W_{t}-\ln W_{0}\right) \times 100 / t$; where $W_{t}$ and $W_{0}$ were final and initial sea cucumber weights respectively; $t$ was duration of experiment in days.

\section{Lysozyme activity}

Lysozyme activity was measured by a blank control method using a LSZ detection kit (Nanjing Jiancheng Bioengineering Institute, China). The increase in the transmittance of the sample at $530 \mathrm{~nm}$ was determined after $15 \mathrm{~min}$ of incubation at $37^{\circ} \mathrm{C}$. One unit of LSZ activity was defined as the amount of enzyme causing a reduction in absorbance of 0.001 per min.

\section{Superoxide dismutase activity}

Superoxide dismutase was measured by its ability to inhibit superoxide anions generated by xanthine and the xanthine oxidase reaction system with the SOD assay kit (Nanjing Jiancheng Bioengineering Institute, China). The optical density was measured at $550 \mathrm{~nm}$. One unit of SOD was defined as the amount required for inhibiting the rate of xanthine reduction by $50 \%$ in a $1-\mathrm{ml}$ reaction system.

\section{Alkaline phosphatase activity}

Alkaline phosphatase activity was determined by using disodium phenyl phosphate as a substrate with a chemical detection kit (Nanjing Jiancheng, Bioengineering Institute, China). The unit definitions of AKP enzymatic activity correspond to the degradation of $1 \mathrm{mg}$ phenol per $100 \mathrm{ml}$ serum at $37{ }^{\circ} \mathrm{C}$ within $15 \mathrm{~min}$. 
Content of complement component 3 (C3)

The content of $\mathrm{C} 3$ was measured using a detection kit made by Zhejiang Elikan Biological Technology. The increase in the absorbance of the sample at $340 \mathrm{~nm}$ was determined after 10 min of incubation at $37^{\circ} \mathrm{C}$. The content of $\mathrm{C} 3$ was proportional to the amount of added antibody.

\section{Vibrio splendidus challenge}

The virulent strain was originally isolated from sea cucumbers diagnosed with skin ulceration disease, which were provided by Yellow-sea Fishery Research Institute, Chinese Academy of Fishery Sciences (Qingdao, China) [20]. The LD50 for 7 days determined before challenge was $5 \times 10^{8} \mathrm{CFU} / \mathrm{ml}$. V. splendidus was grown in tryptic soy broth (TSB) medium with $1.5 \% \mathrm{NaCl}$ at $28{ }^{\circ} \mathrm{C}$ for $24 \mathrm{~h}$, and then was adjusted to $10^{9} \mathrm{CFU} / \mathrm{ml}$. At the end of the feeding trial, 12 sea cucumbers from each group were injected twice with $0.1 \mathrm{ml}$ live $V$. splendidus. Mortality was monitored for 14 days.

\section{Statistical analysis}

All statistical analyses were performed with SPSS version 17.0. The results were presented as mean \pm SE (standard error of the means). Data were analyzed by one-way analysis of variance (ANOVA). When overall differences were significant at less than the $5 \%$ level, Tukey's multiple range tests were used to compare the means among individual treatments.

\section{Results}

Growth performance

After 4 weeks of feeding, the SGRs of the sea cucumbers were significantly affected by the different dietary supplements (Fig. 1). There were no significant differences between group $2(0.34 \%)$ and the control $(0.31 \%)$ $(P>0.05)$, other groups had significantly higher SGRs compared with the control $(P<0.05)$, especially group 6 $(0.97 \%)$.

\section{LSZ activity}

After feeding for 28 days, LSZ activity in the sea cucumbers had increased noticeably with different dietary supplements (Fig. 2a). The sea cucumbers fed a diet supplemented with (APS + TPS) microcapsules $+B$. subtilis exhibited the highest LSZ activity on the 7th day $(P<0.05)$, at $246 \mathrm{U} / \mathrm{ml}$, but there was no significant

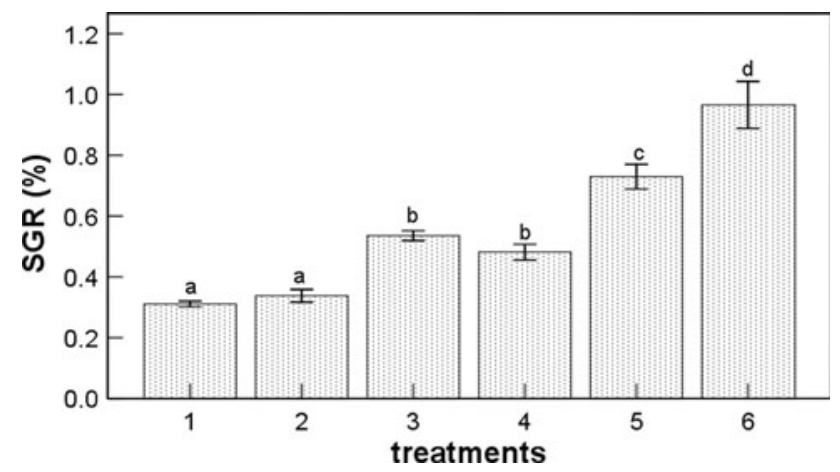

Fig. 1 Effect of different treatments on the growth of Apostichopus japonicus. 1 control, 2 blank microcapsule, 3 Astragalus polysaccharide (APS) microcapsule, 4 tuckahoe (TPS) microcapsule, 5 APS + TPS microcapsule, 6 (APS + TPS) microcapsule + Bacillus subtilis. Values are means and standard errors of three replicates (mean $\pm \mathrm{SE} ; n=3$ ). Treatments with different letters are significantly different $(P<0.05)$

difference compared with group $5(P>0.05)$. The activity reduced with the time, being lower on the 14 th and 28 th days.

\section{SOD activity}

From Fig. $2 b$ we can see that the SOD activity of sea cucumbers fed a diet supplemented with (APS + TPS) microcapsules $+B$. subtilis was at its highest $(P<0.05)$ on the 14th day, up to $91.7 \mathrm{U} / \mathrm{ml}$, and there were irregular changes among the other groups. However, as time progressed, the activities decreased, and there were no significant differences between groups $(P>0.05)$.

AKP activity

The AKP activity value in the coelomic fluid of sea cucumbers is shown in Fig. 2c. Dietary supplementation with (APS + TPS) microcapsules $+B$. subtilis significantly influenced activity, and achieved the highest value (273.8 nkat/l) on the 21st day. This was significant compared with the control $(P<0.05)$. Group 5, fed a diet supplemented with (APS + TPS) microcapsules, showed an obvious advantage, increasing to $234.9 \mathrm{nkat} / \mathrm{l}$ on the 21 st day $(P<0.05)$. Apart from groups 5 and 6 , there were no significant increases between other groups and the control $(P>0.05)$.

\section{C3 content}

C3 content was significantly affected by different dietary supplements, and the result is shown in Fig. 2d. The combination of (APS + TPS) microcapsules and B. subtilis gave the best effect, which was $0.17 \mathrm{~g} / 1$ on the $21 \mathrm{st}$ day $(P<0.05)$, but the control group produced the lowest value. 

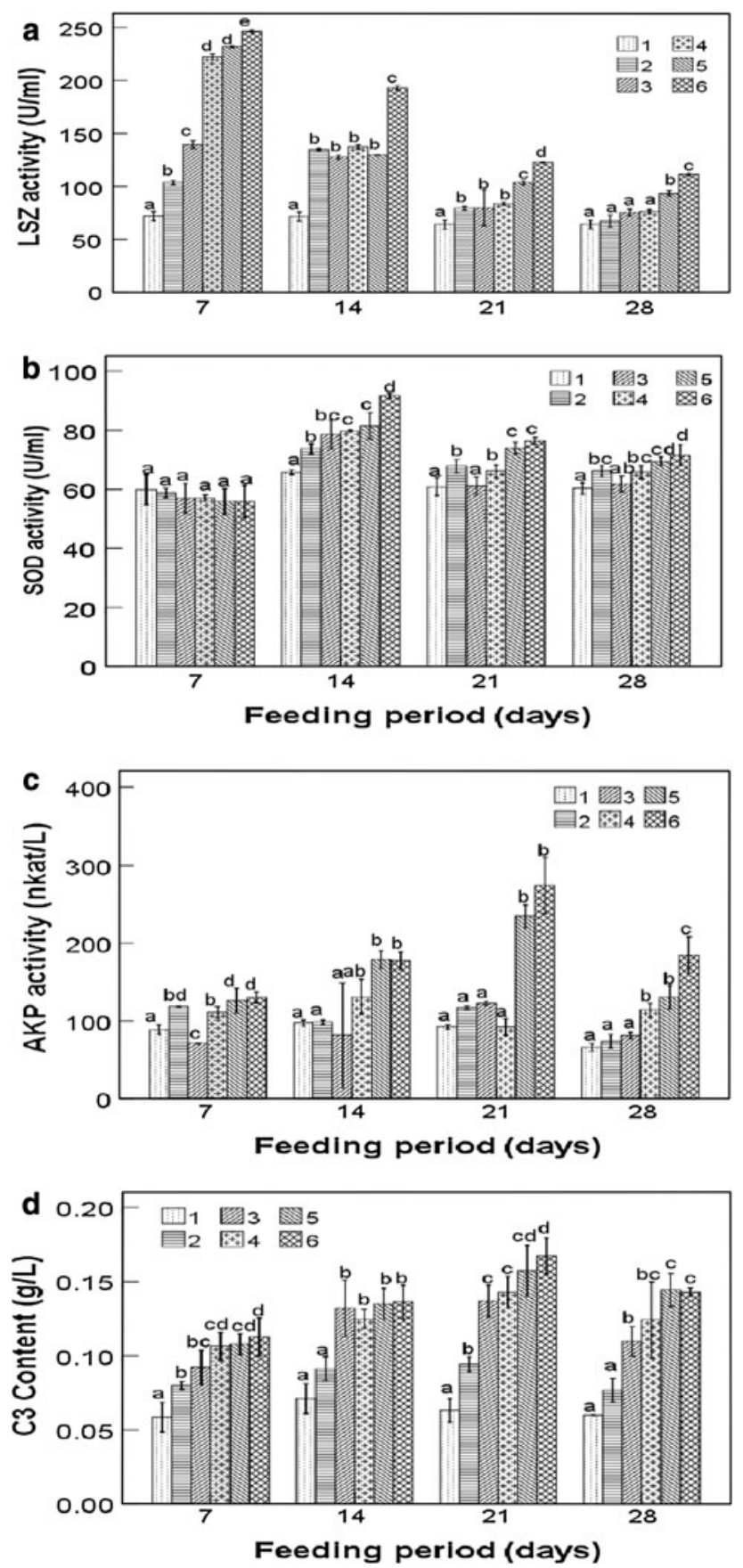

Fig. 2 Lysozyme activity (a), superoxide dismutase activity(b), alkaline phosphatase activity(c), and complement 3 content (d) of Apostichopus japonicus fed with different diets for 4 weeks. 1 control, 2 blank microcapsule, 3 Astragalus polysaccharide (APS) microcapsule, 4 tuckahoe (TPS) microcapsule, 5 APS + TPS microcapsule, 6 (APS + TPS) microcapsule + Bacillus subtilis. Values are means and standard errors of three replicates (mean \pm SE; $n=3$ ). Treatments with different letters are significantly different $(P<0.05)$

\section{Vibrio splendidus challenge}

The cumulative mortality rate within 14 days of sea cucumbers fed with (APS + TPS) microcapsules $+B$.

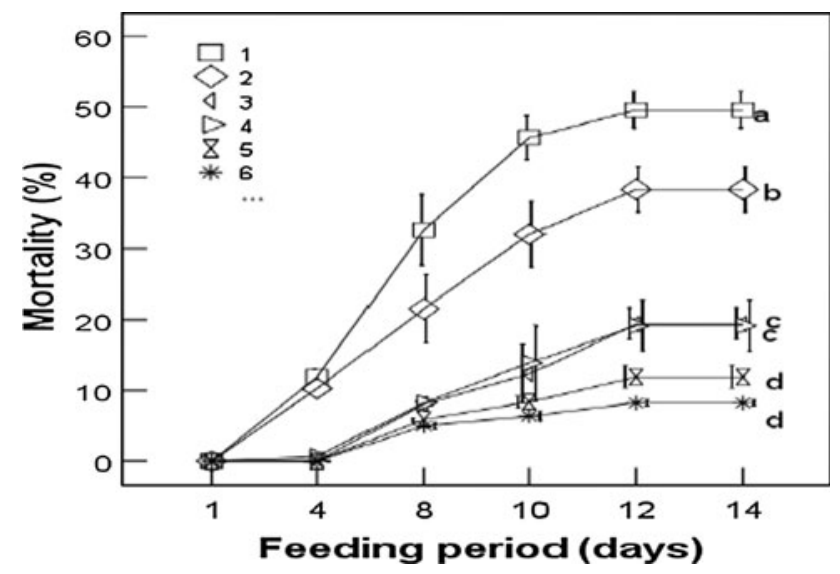

Fig. 3 Cumulative morbidity during a 14-day Vibrio splendidus challenge of Apostichopus japonicus fed with different diets. 1 control, 2 blank microcapsule, 3 Astragalus polysaccharide (APS) microcapsule, 4 tuckahoe (TPS) microcapsule, 5 APS + TPS microcapsule, 6 (APS + TPS) microcapsule + Bacillus subtilis. Values are means and standard errors of three replicates (mean $\pm \mathrm{SE} ; n=3$ ). Treatments with different letters are significantly different $(P<0.05)$

subtilis was only $8.3 \%$, which was significantly lower than that of sea cucumbers fed with the control diet $(49.6 \%)$ $(P<0.05)$. Death of sea cucumbers fed with the control diet was relatively earlier than in other groups (Fig. 3). The challenge test showed that oral administration of (APS + TPS) microcapsules $+B$. subtilis for 4 weeks significantly enhanced protection against $V$. splendidus infection.

\section{Discussion}

Growth performance

The effects of Chinese herbs and their polysaccharides have been studied before, successfully used in aquaculture and have been verified to affect growth and non-specific immunity [9, 14, 21, 22]. Wang et al. [14] showed that supplements of $3 \%$ conventional fine powder (CP) or superfine powder (SP) of Astragalus membranaceus root or $0.3 \%$ APS, given over a period of 60 days could enhance the immune responses of A. japonicus. Some studies showed that feeding Chinese herb polysaccharides or their combinations could improve the growth of animals [18, 23]. Gu et al. [15] demonstrated that dietary $\beta$-glucan, manna oligosaccharide (MOS) and their combinations for 4 weeks had obvious effects on the growth performance of the sea cucumber A. japonicus. However, little work has been undertaken to study the application of microcapsule polysaccharides in aquaculture; neither the application of microencapsulation technology products in aquaculture, nor the effect on A. japonicus. In the present work, it is suggested that feeding combinations of (APS + TPS) 
microcapsules and $B$. subtilis significantly increased the SGR of sea cucumbers $(P<0.05)$. It reached $0.97 \%$, higher than (APS + TPS) microcapsules or APS microcapsules or TPS microcapsules. It could be suggested that among the different active substances in Chinese herbs there are significant synergies, for example between APS and TPS, and the B. subtilis could further enhance their effect, perhaps due to their regulatory role in the environment or ecology.

Probiotics (such as Bacillus), defined as micro-organisms whose products are healthy to the host, are known for their antagonism towards pathogens, enhancement of growth, immune response and feeding efficiency, and improvement of micro-flora balance in humans and animals [24-27]. The genus Bacillus has been used extensively as a feed additive that can be resistant to high temperature and high pressure [28-30]. Some studies showed that dietary B. subtilis can improve the growth of aquatic animals, but suitable doses in the diet are needed [31-33], based on differences in strains, animals (species and sizes) and experimental conditions [13, 17, 34, 35]. Zhang et al. [17] demonstrated that a commercial B. subtilis could significantly increase growth of $A$. japonicus on a $1.82 \times 10^{7} \mathrm{CFU} / \mathrm{g}$ diet for 56 days. Compared to those reports, in the present study, the dietary level of $B$. subtilis was near $2 \times 10^{7} \mathrm{CFU} / \mathrm{g}$, which could enhance the synergistic effect on the SGR of A. japonicus. But Zhao et al. [13] showed that a dietary level of B. subtilis T13 at a much higher level $\left(10^{9} \mathrm{CFU} / \mathrm{g}\right)$ significantly improved the SGR of sea cucumbers.

Moreover, in the current study, APS and TPS microcapsules could be mixed directly with the basal diet and then the mixture could be sprayed evenly into aquaria, to decrease the loss of polysaccharides. Microcapsules are a new dosage form of immune-enhancement agents, applied in aquaculture, especially suitable for animals with certain feeding characteristics, such as sea cucumbers.

\section{Immune response}

Modulation of the immune system is one of the ordinary benefits of the Chinese herb active substances and probiotics. The present study also demonstrated that APS, TPS and $B$. subtilis could significantly stimulate the immune response of sea cucumbers. As we all know, sea cucumbers lack an adaptive immune system; their humoral immune responses are the second line of defense against infections and injuries [5, 36]. And in the humoral immune response, lysosomal enzymes, superoxide dismutase, alkaline phosphatase, and complement together participate in the destruction of external substances, so that they can play a protective role. The single Chinese herb of $A$. membranaceus or APS has been shown to increase lysozyme activity in fish blood and in sea cucumbers [8, 9, 14, 19]. However, the combination of polysaccharides and $B$. subtilis has not been previously reported, or the microcapsule technology. In the present study, APS microcapsules or TPS microcapsules could increase lysozyme values $(P<0.05)$ during the experiment to different extents; on the 7 th day the lysozyme values reached the highest point, showing a similar course over time as groups 5 and 6 . Moreover, combination of APS and TPS microcapsules, and combination of (APS + TPS) microcapsules and $B$. subtilis indicated a stronger effect; the combined effect among different Chinese herbs could have a complementary synergy, which is usually called compatibility. Furthermore, the role of $B$. subtilis in regulating microecological balance and water quality indirectly enhanced the growth and immune effect of sea cucumber, as seen in the performance of LSZ activity.

SOD catalyses the dismutation of the extra bactericidal highly reactive $\mathrm{O}^{2-}$ to $\mathrm{O}_{2}$ and less reactive $\mathrm{H}_{2} \mathrm{O}_{2}$, and it is an important component of the antioxidant defense system of the organism [37, 38]. In our laboratory, the tests by injecting APS and TPS into A. japonicus suggested that APS and TPS could significantly increase SOD activity in the coelomic fluid of the sea cucumber, and enhance antioxidant capacity, which could be related to the role that flavonoids and saponins play in A. membranaceus to eliminate oxygen free radicals. The effect of dietary administration of APS, TPS, and B. subtilis on SOD activity of the sea cucumber had different results in the present study. And a significant difference was observed in group 6 on the 14th day; $91.7 \mathrm{U} / \mathrm{ml}(P<0.05)$. Other treatments also gave higher values on the 14th day; there was a rise-drop trend between the time and the effect. This could be associated with the structure of the polysaccharide and immunomodulatory mechanisms, as well as the immune system of sea cucumbers. The results of the present study confirmed that the APS and TPS microcapsules could be fed directly to the sea cucumbers, the size of the microcapsules were right for them, while at the same time polysaccharide loss was reduced in the seawater; the microcapsule in actual application is suitable for breeding and easily accepted by farmers.

Alkaline phosphatase is a marker enzyme of the phagocytes lysosomal, especially in the mollusks. Sun et al. [39] pointed out that AKP in the sea cucumber immune system plays an important role in the process of swallow the foreign substances. The experimental results indicated that AKP activity showed a sensitive response to the combination of (APS + TPS) microcapsules and $B$. subtilis, reaching the highest value $(273.8$ nkat/l) on the $21 \mathrm{st}$ day $(P<0.05)$. However, the activity had decreased by the 28 th day. So we deduced that there was a certain degree of correlation between the dietary time and activity. 
At present the complement system is a highly sophisticated defense system against common pathogens acting in the innate immunity of invertebrates and vertebrates, which is induced by antigen-antibody interactions in the traditional pathway. As a central component in the complement system, component 3 is an intermediary between the innate and the adaptive immune systems $[40,41]$. During the past few years, the homologs of C3 have been identified, from higher vertebrates to lower protostomes including human, fish, amphioxus, sea squirt, sea urchin, horseshoe crab, coral, and sea anemone [42-44]. Past studies have shown that there are analogs of complement in Asterias forbesi, and in its coelomic cells there were $\mathrm{C} 3 \mathrm{~b}$ and $\mathrm{C} 3 \mathrm{bi}$ complement receptors [45, 46]. Using enzyme-linked chemiluminescence immune detection (chemiluminescent immunoassay, CLIA), they found complement analogs in the coelomic fluid of the sea cucumber: the content of C3 was $6.58( \pm 1.4) \mu \mathrm{g} / \mathrm{ml}$, and C4 was $0.67( \pm 0.3) \mu \mathrm{g} / \mathrm{ml}$. This is an important observation about A. japonicus complement, and may provide a theoretical basis for the development of new immunostimulants [47, 48]. Zhou et al. [49] studied the molecular characterization and expression analysis of $\mathrm{C} 3$ in the sea cucumber (A. japonicus), it was suggested that $\mathrm{AjC} 3-2$ and $\mathrm{AjC} 3$ genes play a pivotal role in immune responses to bacterial infection in sea cucumbers. The results showed that complement of $A$. japonicus played an important role in the immune system. In the present study, C3 content was highest on the 21 st day in group 6 , reaching $0.17 \mathrm{~g} / \mathrm{l}$; it was significantly different to the control group and groups 2,3 , and $4(P<0.05)$. Sea cucumber C3 needs further research. C3 content and activities of other enzymes reached their highest values at different times. This may be related to the regulation mechanism of immunostimulants, since different immune indices have different responses.

\section{Challenge assay}

The challenge assay is the direct way to reflect the effect of dietary immunostimulants. $V$. splendidus is the pathogen causing skin ulcer syndrome, which can cause serious harm to sea cucumber aquaculture. Dong et al. [3] have shown that exposure of $A$. japonicus to $V$. splendidus at a concentration of $10^{6}$ cells $/ \mathrm{ml}$ for 6 days could result in the occurrence of disease. To determine the efficacy of different dietary supplements, it has been shown that the survival rate of $A$. japonicus and its resistance to $V$. splendidus could be enhanced by administration of Astragalus and APS [14]. The present study showed that the oral administration of (APS + TPS) microcapsules and B. subtilis reduced the mortality of sea cucumber after being challenged by $V$. splendidus. The improved resistance of sea cucumbers may be partly attributable to the increased activities of different enzymes. There was significant difference between blank microcapsules and the control, which could be due to the effects of sodium alginate. Sodium alginate is alginate extracted from seaweed, and other factors could cause the errors. The resistance of sea cucumbers was further improved after adding APS and other microcapsules, however, there was no significant difference between groups 5 and 6 , but group 6 also reflected an increase under the action of $B$. subtilis, that probably was due to the improvement of water quality. Zhao et al. [13] reported that A. japonicus fed with probiotic $B$. subtilis T13 show significant improved resistance against $V$. splendidus.

As we all know, the role of probiotics could selectively stimulate the growth and activity of one or more bacteria, and so result in beneficial or harmful effects on the host $[24,50]$. This may be the reason that APS, TPS, and $B$. subtilis could improve the resistance to disease of sea cucumbers in the present study. Polysaccharides could play the role of probiotics and stimulate the secretion of cytokines to improve immune function and increase glucose tolerance.

Acknowledgments We would like to thank the Fund Project of Shandong Province modern agricultural technology system for sea cucumber innovation team; shandong province policy project (No. 2012YD10016); marine public research fund project (No. 201305005). We also thank Drs Diao and Chen for their contribution on checking the manuscript.

Open Access This article is distributed under the terms of the Creative Commons Attribution License which permits any use, distribution, and reproduction in any medium, provided the original author(s) and the source are credited.

\section{References}

1. Reilly A, Kaferstein F (1997) Food safety hazards and the application of the principles of the hazard analysis and critical control point (HACCP) system for their control in aquaculture production. Aquac Res 28:735-752

2. Bachère E (2003) Anti-infectious immune effectors in marine invertebrates: potential tools for disease control in larviculture. Aquaculture 227:427-438

3. Dong Y, Deng H, Sui XL, Song L (2005) Ulcer disease of farmed sea cucumber (Apostichopus japonicus). Fish Sci (China) 24:4-6 (in Chinese, with English abstract)

4. Deng H, He CB, Zhou ZC, Liu C, Tan KF, Wang NB, Jiang B (2009) Isolation and pathogenicity of pathogens from skin ulceration disease and viscera ejection syndrome of the sea cucumber Apostichopus japonicus. Aquaculture 287:18-27

5. Sun YX, Qang JQ, Wang TT, Xue JP, Liu G, You JH, Xu YP (2007) A review: defense mechanism in sea cucumber. Fish Sci (China) 26:358-361 (in Chinese, with English abstract)

6. Bedir E, Pugh N, Calis I, Pasco DS, Khan IA (2000) Immunostimulatory effects of cycloartane-type triterpene glycosides from Astragalus species. Biol Pharm Bull 23:834-837 
7. Tan BK, Vanitha J (2004) Immunomodulatory and antimicrobial effects of some traditional Chinese medicinal herbs: a review. Curr Med Chem 11:1423-1430

8. Chansue N, Ponpornpisit A, Endo M, Sakai M, Satoshi Y (2000) Improved immunity of tilapia Oreochromis niloticus by CUP III, a herb medicine. Fish Pathol 35:89-90

9. Jian JC, Wu ZH (2004) Influences of traditional Chinese medicine on non-specific immunity of Jian carp (Cyprinus carpio var. Jian). Fish Shellfish Immunol 16:185-191

10. Shao BM, Xu W, Dai H, Tu P, Li Z, Gao XM (2004) A study on the immune receptors for polysaccharides from the roots of Astragalus membranaceus, a Chinese medicinal herb. Biochem Biophys Res Commun 320:1103-1111

11. Seguin-Devaux C, Hanriot D, Dailloux M, Latger-Cannard V, Zannad F, Mertes PM, Longrosis D, Devaux Y (2005) Retinoic acid amplifies the host immune response to LPS through increased $\mathrm{T}$ lymphocytes number and LPS binding protein expression. Mol Cell Endocrinol 245:67-76

12. Dalmo RA, Bøgwald J (2008) $\beta$-glucans as conductors of immune symphonies. Fish Shellfish Immunol 5:384-396

13. Zhao YC, Zhang WB, Xu W, Mai KS, Zhang YJ, Liufu ZG (2012) Effects of potential probiotic Bacillus subtilis T13 on growth, immunity and disease resistance against Vibrio splendidus infection in juvenile sea cucumber Apostichopus japonicus. Fish Shellfish Immunol 32:750-755

14. Wang TT, Sun YX, Jin LJ, Xu YP, Wang L, Ren TJ, Wang KL (2009) Enhancement of non-specific immune response in sea cucumber (Apostichopus japonicus) by Astragalus membranaceus and its polysaccharides. Fish Shellfish Immunol 27:757-762

15. Gu M, Ma HM, Mai KS, Zhang WB, Bai N, Wang XJ (2011) Effects of dietary $\beta$-glucan, mannan oligosaccharide and their combinations on growth performance, immunity and resistance against Vibrio splendidus of sea cucumber, Apostichopus japonicus. Fish Shellfish Immunol 31:303-309

16. Ortuño J, Cuesta A, Esteban A, Meseguer J (2001) Effect of oral administration of high vitamin $\mathrm{C}$ and $\mathrm{E}$ dosages on the gilthead sea bream (Sparus aurata L.) innate immune system. Vet Immunol Immunopathol 79:167-180

17. Zhang Q, Ma HM, Mai KS, Zhang WB, Liufu ZG, Xu W (2010) Interaction of dietary Bacillus subtilis and fructooligo saccharide on the growth performance, nonspecific immunity of sea cucumber, Apostichopus japonicus. Fish Shellfish Immunol 29:204-211

18. Selvaraj V, Sampath K, Sekar V (2006) Adjuvant and immunostimulatory effects of $\beta$-glucan administration in combination with lipopolysaccharide enhances survival and some immune parameters in carp challenged with Aeromonas hydrophila. Vet Immun Immunopathol 114:15-24

19. Fan Y, Wang SX, Ye HB, Xu L, Zhu AC, Yang XS, Li TB (2010) Effects of polysaccharides from Astragalus membranaceus on non-specific immune in sea cucumber Apostichopus japonicus. Fish Sci (China) 29:321-324 (in Chinese, with English abstract)

20. Zhang CY, Wang YG, Rong XJ (2006) Isolation and identification of causative pathogen for skin ulcerative syndrome in Apostichopus japonicus. J Fish China 30:106-118 (in Chinese, with English abstract)

21. Yin GJ, Jeney G, Racz T, Xu P, Jun X, Jeney Z (2006) Effect of two Chinese herbs (Astragalus radix and Scutellaria radix) on nonspecific immune response of tilapia, Oreochromis niloticus. Aquaculture 253:39-47

22. Huang XX, Zhou HQ, Zhang H (2006) The effect of Sargassum fusiforme polysaccharide extracts on vibriosis resistance and immune activity of the shrimp, Fenneropenaeus chinensis. Fish Shellfish Immunol 20:750-757

23. Gu M, Mai KS Ma HM, Zhang WB, Ai QH, Wang XJ, Bai N (2010) Immune response of sea cucumber Apostichopus japonicus coelomocytes to several immunostimulants in vitro. Aquaculture 306:49-56

24. Suzer C, Çoban D, Kamaci HO, Saka Ş, Firat K, Özge Otgucuoğlu, Küçüksari H (2008) Lactobacillus spp. bacteria as probiotics in gilthead sea bream (Sparus aurata, L.) larvae: effects on growth performance and digestive enzyme activities. Aquaculture 280:140-145

25. Wang YB, Li JR, Lin JD (2008) Probiotics in aquaculture: challenges and outlook. Aquaculture 281:1-4

26. Gatesoupe FJ (1999) The use of probiotics in aquaculture. Aquaculture 180:147-165

27. Li JQ, Tan BP, Mai KS (2009) Dietary probiotic Bacillus OJ and isomalto oligosaccharides influence the intestine microbial populations, immune responses and resistance to white spot syndrome virus in shrimp (Litopenaeus vannamei). Aquaculture 291:35-40

28. Rengpipat S, Rukpratanporn S, Piyatiratitivorakul S, Menasaveta $P$ (2000) Immunity enhancement in black tiger shrimp (Penaeus monodon) by a probiont bacterium (Bacillus S11). Aquaculture 191:271-288

29. Rengpipat S, Phianphak W, Piyatiratitivorakul S, Menasveta P (1998) Effects of a probiotic bacterium on black tigers shrimp Penaeus monodon survival and growth. Aquaculture 167:301-313

30. Ochoa-Solano JL, Olmos-Soto J (2006) The functional property of Bacillus for shrimp feeds. Food Microbiol 23:519-525

31. Liu CH, Chiu CS, Ho PL, Wang SW (2009) Improvement in the growth performance of white shrimp, Litopenaeus vannamei, by a protease-producing probiotic, Bacillus subtilis E20, from natto. J Appl Microbiol 107:1031-1041

32. Tseng DY, Ho PL, Huang SY, Cheng SC, Shiu YL, Chiu CS, Liu CH (2009) Enhancement of immunity and disease resistance in the white shrimp, Litopenaeus vannamei, by the probiotic, Bacillus subtilis E20. Fish Shellfish Immunol 26:339-344

33. Aly SM, Mohamed MF, John G (2008) Effect of probiotics on the survival, growth and challenge infection in Tilapia nilotica (Oreochromis niloticus). Aquac Res 39:647-656

34. Nayak SK (2010) Probiotics and immunity: a fish perspective. Fish Shellfish Immunol 29:2-14

35. Sun YZ, Yang HL, Ma RL, Lin WY (2010) Probiotic applications of two dominant gut Bacillus strains with antagonistic activity improved the growth performance and immune responses of grouper Epinephelus coioides. Fish Shellfish Immunol 29:803-809

36. Canicatti C, Parrinello N (1985) Hemaglutinin and hemolysin level in coelomic fluid from Holothuria polii (Echinodermata) following sheep erythrocyte injection. Biol Bull 168:175-182

37. Fu LL, Shuai JB, Xu ZR, Li JR, Li WF (2010) Immune responses of Fenneropenaeus chinensis against white spot syndrome virus after oral delivery of VP28 using Bacillus subtilis as vehicles. Fish Shellfish Immunol 28:49-55

38. Castex M, Lemaire P, Wabete N, Chim L (2010) Effect of probiotic Pediococcus acidilactici on antioxidant defences and oxidative stress of Litopenaeus stylirostris under Vibrio nigripulchritudo challenge. Fish Shellfish Immunol 28:622-631

39. Sun YX, Jin LJ, Wang TT, Xue JP, Liu G, Li XY, You JS, Li SY, Xu YP (2008) Polysaccharides from Astragalus membranaceus promote phagocytosis and superoxide anion $\left(\mathrm{O}_{2}{ }^{-}\right)$production by coelomocytes from sea cucumber Apostichopus japonicus in vitro. Comp Biochem Phys Part C 147:293-298

40. Dunkelberger JR, Song WC (2010) Complement and its role in innate and adaptive immune responses. Cell Res 20:34-50

41. Fujita T (2002) Evolution of the lectin-complement pathway and its role in innate immunity. Nat Rev Immunol 2:346-353

42. Boshra H, Li J, Sunyer JO (2006) Recent advances on the complement system of teleost fish. Fish Shellfish Immunol 20:239-262 
43. Dishaw LJ, Smith SL, Bigger CH (2005) Characterization of a C3-like cDNA in a coral: phylogenetic implications. Immunogenetics 57:535-548

44. Fujito NT, Sugimoto S, Nonaka M (2010) Evolution of thioestercontaining proteins revealed by cloning and characterization of their genes from a cnidarian sea anemone, Haliplanella lineate. Dev Comp Immunol 34:775-784

45. Berthuessen K, Seljelid R (1982) Receptors for complement on echinoid phagocytes. The opsonic effect of vertebrae sera on echinoid phagocytosis. Dev Comp Immunol 6:423-431

46. Leonard LA, Strandberg JD, Winkelstein JA (1990) Complement-like activity in the sea star, Asterias forbesi. Dev Comp Immunol 14:19-30

47. Zhang F, Gong J, Wang HF, Zhao X, Li XY (2007) The detection of complement analogues $\mathrm{AjC} 3$, and $\mathrm{AjC} 4$ in the sea cucumber
Apostichopus japonicus. J Dalian Fish Univ 22:246-248 (in Chinese, with English abstract)

48. Zhang F, Wang HF, Gong J, Chang SJ (2007) Detection method of complement analogues of coelomic fluid in Apostichopus japonicus by chemiluminescent immunoassay. J Nuclear Agric Sci (JNAS) 21:413-416 (in Chinese, with English abstract)

49. Zhou ZC, Sun DP, Yang AF, Dong Y, Chen Zh, Wang XY, Guan XY, Jiang B, Wang B (2011) Molecular characterization and expression analysis of a complement component 3 in the sea cucumber (Apostichopus japonicus). Fish Shellfish Immunol 31:540-547

50. Vine N, Leukes W, Kaiser H (2006) Probiotics in marine larviculture. FEMS Microbiol Rev 30:404-427 